\title{
Miranda
}

Revue pluridisciplinaire du monde anglophone /

Multidisciplinary peer-reviewed journal on the English-

speaking world

$21 \mid 2020$

Modernism and the Obscene

Monica Latham et Nathalie Collé (ed), Mark SaFranko: The Creative Itineraries of a "Renaissance Man"

\section{Brigitte Friant-Kessler}

\section{OpenEdition}

\section{Journals}

Electronic version

URL: http://journals.openedition.org/miranda/30407

DOI: 10.4000/miranda.30407

ISSN: 2108-6559

\section{Publisher}

Université Toulouse - Jean Jaurès

\section{Electronic reference}

Brigitte Friant-Kessler, "Monica Latham et Nathalie Collé (ed), Mark SaFranko: The Creative Itineraries of a "Renaissance Man"'", Miranda [Online], 21 | 2020, Online since 13 October 2020, connection on 16 February 2021. URL: http://journals.openedition.org/miranda/30407; DOI: https://doi.org/10.4000/ miranda.30407

This text was automatically generated on 16 February 2021.

\section{cc)}

Miranda is licensed under a Creative Commons Attribution-NonCommercial-NoDerivatives 4.0

International License. 


\section{Monica Latham et Nathalie Collé (ed), Mark SaFranko: The Creative} Itineraries of a "Renaissance Man"

Brigitte Friant-Kessler

\section{REFERENCES}

Monica Latham et Nathalie Collé (ed), Mark SaFranko: The Creative Itineraries of a "Renaissance Man",Presses Universitaires de Nancy, 2019. ISBN 978-2-8143-0537-3 


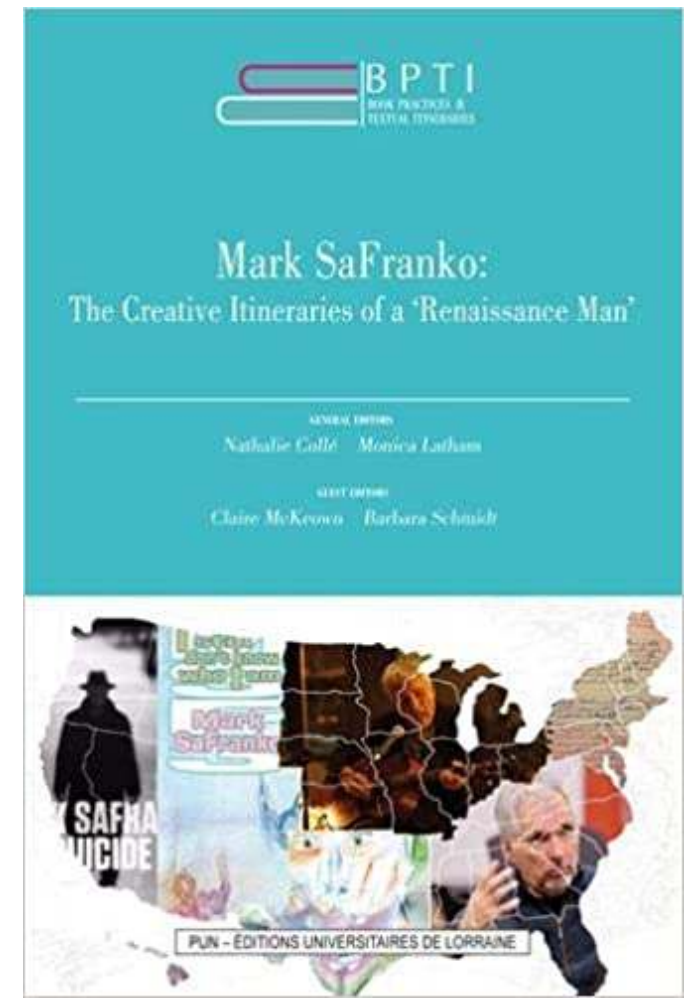

$1 \mathrm{Au}$ commencement, une résidence d'artiste (ARIEL) et un projet pédagogique interdisciplinaire et innovant, porté par Barbara Schmidt et Céline Sabiron, au sein de l'université de Lorraine, en collaboration avec les chercheurs d'IDEA, ce qui permet de relier la recherche, des pratiques créatives et l'enseignement des langues vivantes (niveau Master) à la présence d'un auteur vivant. Autour de ce projet riche et ambitieux gravitent ensuite des activités, des événements culturels et scientifiques et des publications (version papier et version électronique). C'est donc à un ensemble de formats et de publications satellitaires que se rattache cet ouvrage (PUN-EDULOR) de la collection BPTI, «Book Practices and Textual Itineraries », dirigée par Nathalie Collé et Monica Latham.

2 Miranda a également déjà publié trois entretiens avec Mark SaFranko dans Ariel's Corner:

3 Armand, Claudine. « Piecing the Puzzle Together: An Interview with Writer and Artist Mark SaFranko. » Miranda [Online], vol. 18, 2019.

4 Bak, John S. "In Interview with American Playwright Mark SaFranko.» Miranda [Online], vol. 18, 2019.

5 Heberlé, Jean-Philippe. « An Interview with Mark SaFranko: The Songwriter. » Miranda [Online], vol. 18, 2019.

Dans le volume BPTI 11 'Mark SaFranko: Itineraries of a Renaissance Man' sont consignés et retranscrits des conférences, des entretiens avec des chercheurs de l'université de Lorraine, mais également des dialogues avec des étudiants en Master qui ont participé à la mise en scène et mise en voix de textes de SaFranko, sous la houlette de l'écrivain. Avec ce volume, nous entrons dans les coulisses de l'écriture et de la créativité d'un écrivain. L'ensemble est à la fois hétérogène par les formats et charpenté par la volonté de faire découvrir un auteur américain finalement assez peu connu. Le tout a résolument des allures de making of : on s'attache à montrer toutes les 
coutures qui d'habitude restent plutôt discrètes pour donner une visibilité au processus d'écriture et de fabrication des textes, notamment dans leurs états de notes ou encore de brouillons successifs (49-92) reproduits. Le volume témoigne aussi des étapes (conférences, séminaires, concerts, expositions) de la résidence ARIEL, sigle dont la signification 'Auteur en Résidence Internationale en Lorraine' est redonnée à plusieurs endroits de l'ouvrage.

7 Itinéraires d'un enfant peu gâté ('I come from a blue collar background' comme le rappelle souvent SaFranko lui-même), les chemins de traverse de Mark SaFranko sont nombreux et les passerelles entre différentes formes d'art multiples: romancier, nouvelliste (Raymond Carver n'est jamais très loin chez SaFranko), auteur de pièces de théâtre, poète, compositeur et musicien accompli (Voir son site personnel : https:// marksafranko.com). Sa créativité se déploie en effet dans différents médias et quelquefois jusqu'au bout de pinceaux qui lui permettent de jouer avec la fluidité de l'aquarelle. Inutile de viser l'exhaustivité, elle n'est pas au cœur du projet, ni d'ailleurs dans l'esprit de SaFranko dont le morceau musical I Still Don't Know Who I am (reproduit page 258) figure dans l'album Best of 3 mais sert aussi de titre pour un autre album, montrant ainsi que la formule résume bien une personnalité en perpétuelle quête d'identité. On peut écouter le morceau ici: https://www.youtube.com/watch? $\mathrm{v}=$ =dxf5GYuXFo\&list=PLhUu-biRVwigUKu718zy-3X8w6nG_QJJ9\&index=85

8 Son expression et son style mêlent les jeux proustiens du 'je' auctorial à la rugosité d'une langue célinienne. Écrivain autodidacte, journaliste aussi, SaFranko est souvent décrit comme le "plus Frenchy des Américains", il ne cache pas son immense admiration pour l'auteur du Voyage au bout de la nuit et pour Georges Simenon : 'I stole from Celine' (112). Parmi les préférés de sa bibliothèque il y a d'autres grandes figures comme Dostoïevsky, Camus dont il aime surtout L'étranger et le Philippe Djian de $37^{\circ} 2$ le matin. Américain sans doute, SaFranko porte néanmoins en lui les gènes de cette Amérique issue de l'immigration de l'Europe de l'Est et nourrit ainsi une irrésistible attraction vers la chose européenne. La France tient une place à part dans son cœur. De son propre aveu, sa carrière a d'ailleurs véritablement pris un élan depuis l'autre côté de l'Atlantique (France, Angleterre) et non sur les terres de Hawthorne, ou d'Hemingway, ni même de Dan Fante dont il était un ami et qui dit de SaFranko «Je crois bien qu'à choisir, ce gars-là préférerait écrire que respirer » (Épigraphe de la pièce Minable, première pièce de théâtre publiée en langue française, E-Fractions Editions, 2013). Proche d'un Hubert Selby Jr., Henry Miller ou d'un Charles Bukowski, SaFranko trouve en Europe le terreau qui inspire son écriture classée tantôt néo-beat, tantôt « dirty realism ». L'Europe c'est aussi pour lui un écosystème éditorial et culturel plus propice à la diffusion de ses textes que Harper Collins, du moins à ses débuts. «Mark Safranko, cet écrivain américain emblématique de l'underground new-yorkais, ce genre de type qu'on adore en France. » http://bookalicious.fr/plaid-nouveautes/ (consulté le 22 août 2020)

9 Les librairies où il a signé ses romans en Lorraine dans le cadre de la résidence en sont encore une preuve.

10 L'homme aime à dire qu'il se verrait bien vivre au temps de la Renaissance où l'éclectisme intellectuel et la curiosité des humanistes s'étaient emparés de tout un continent. Il réitère à l'envi que le marché du livre aux États Unis ne respire que par le nom de Stephen King et n'aspire hélas guère à d'autres horizons littéraires. Dans la sous-partie '55 questions to Mark SaFranko', il se prête au jeu d'un portrait chinois 
(245-49) qui livre l'essentiel de sa philosophie de vie. Si rien ne le destinait au métier d'artiste ou d'écrivain, une fois l'obstination de l'écriture installée dans sa tête, l'écriture s'est imposée à lui, plus que lui ne l'avait choisie pour en vivre. Qualifié parfois d'auteur underground, SaFranko dont on découvre au fil des sections du volume les diverses facettes: Authorial Testimonies, Translation, Reception, Portraits of the Artist.

11 On peut relever plusieurs types de contributions : entretiens et dialogues avec l'auteur, témoignages commentés des activités menées avec l'écrivain (séminaires, pratiques théâtrales, traduction) et les transcriptions des conférences, textes d'auteurs, brouillons de textes. La sous-partie intitulée « Portraits of the Artist» rassemble des reproductions d'aquarelles, exposées (nov. 2018 -jan. 2019) au Centre d'Arts Chalon de l'université de Lorraine. Ce sont essentiellement des autoportraits de l'artiste, agrémentés de quelques lignes descriptives. L'ensemble des interventions et entretiens est centré sur SaFranko pour former un portrait kaléidoscopique, ce qui semble bien correspondre à l'homme et à l'artiste-auteur-compositeur-musicien. Il est parfois difficile de se départir d'un sentiment de fragmentation quelque peu trop éclatée à la lecture des différents 'morceaux du puzzle', pour reprendre l'expression employée dans l'entretien réalisé par Claudine Armand. Que retenir de tous ces fragments? Une vie trépidante et bien remplie pour l'auteur qui relate combien il lui a été difficile de percer dans son propre pays. Une écriture compulsive et nécessaire, non pour le public mais pour la survie. Enfin, et surtout, il ressort des différents entretiens que la résidence a permis des collaborations et rencontres chaleureuses et fructueuses, y compris pour l'écrivain qui a rencontré Philippe Claudel et qui a commis une nouvelle dont le sujet, un incident vécu à Nancy durant son séjour, fait aussi l'objet d'une traduction, le tout reproduit en intégralité ici: http://ariel.univ-lorraine.fr/lalorraine-vue-par-les-auteurs-ariel/mark-safranko-an-incident/

ARIEL se perçoit comme un projet pédagogique stimulant et dynamisant pour des étudiants Master dont certains déjà engagés dans la recherche, alors que d'autres ont pu bénéficier de la présence de l'écrivain-dramaturge durant des ateliers de pratiques théâtrales. L'essentiel du volume étant constitué de transcriptions écrites d'entretiens avec l'écrivain en résidence et de communications faites par ce dernier à l'occasion de séminaires ou d'invitations en tant que conférencier, la publication de ces morceaux choisis oscille entre écrit et oral. La voix de SaFranko est lue et vue (50-60), une expérience sensorielle et cognitive qui peut être complétée par des vidéos ou des enregistrements audio pour donner un corps performatif à ces textes. En effet, il est possible d'écouter l'enregistrement de l'interview qui est retranscrit ici : https:// videos.univ-lorraine.fr/index.php?act=view\&id=7377 (consulté 24 août 2020). Le format même des transcriptions d'entretiens induit inévitablement des répétitions, mais les ateliers ont chacun des objectifs spécifiques. On lit avec intérêt l'article de Heather DUERRE HUMANN qui approfondit une partie abordée dans son ouvrage Gender Bending in Detective Fiction. On regrette la qualité de résolution de certaines reproductions $(56-57,75,163,167)$ assez surprenante pour une collection dont les volumes témoignent en général d'une grande attention aux illustrations. Les dimensions des portraits de l'exposition auraient permis de se faire une meilleure idée de la production plastique de l'écrivain. On peut aussi se demander s'il fallait vraiment reproduire en version papier tous les posters, affiches et visuels de communication. Depuis 2018, le projet de ces résidences se poursuit à l'université de Lorraine : on peut en consulter les archives et suivre les différentes activités, qui sont susceptibles d'inspirer d'autres universités 
en France et ailleurs. Il faut saluer ces initiatives qui offrent aux étudiants l'occasion, peut être unique, de côtoyer durant leur formation, ou même de leur vie, un auteurartiste de son vivant (130). Après SaFranko, l'université de Lorraine a accueilli une auteure italienne en 2019. Il faut nourrir l'espoir de voir d'autres auteur-e-s d'autres horizons participer à ce projet de résidence. Longue vie à ARIEL, donc !

INDEX

Keywords: Dirty realism, Neo Beat writer, artist residence, novel, short story, crime fiction, playwright, music, composer, song writer, publishing

Mots-clés: « réalisme sale », écrivain Néo-Beat, résidence d'artiste, roman, nouvelle, fiction policière, dramaturge, musique, auteur-compositeur, monde de l'édition

\section{AUTHORS}

\section{BRIGITTE FRIANT-KESSLER}

Maître de conférences en études anglophones et arts visuels UPHF, Université Polytechnique Hauts de France, Valenciennes bfriantk@uphf.fr 